\title{
A simplified view on entropic gravity
}

\section{Engel Roza}

Philips Research, Eindhoven, The Netherlands (retired)

Stripperwei 1, 5551 ST Valkenswaard, The Netherlands

Email: engel.roza@onsbrabantnet.nl

\section{Summary}

In this article a simplified view is given on Verlinde's entropic gravity. It reveals several shortcomings in the theory. This holds in particular for the inclusion of the dark matter effect in the derived modified expression for the Newtonian gravitational acceleration. Although the merit of the entropic view on gravity is recognized, the proposed explicit axiomatic hypothesis as a basis is criticized. There is no reason why it should be preferred above Einstein's Field Equation.

Keywords: entropic gravity; MOND; dark matter

\section{Introduction}

Entropic gravity is a concept, in 2011 introduced by Erik Verlinde [1]. It aims to explain gravity by inheriting the entropic principles of black holes, such as originally described by Bekenstein [2] and Hawking [3]. Because of its claim that it gives an adequate explanation of the dark matter phenomenon in cosmology, such as empirically described by Milgrom's MOND theory [4], it attracted a lot of interest, even to the extent of a high exposure in public media. Whether the claim is justified still is subject to a scientific debate with pros and cons. One of the problems here is the deciphering of the highly sophisticated scientific language used by Verlinde in the two articles [1,5], in which he describes his theory. It is my aim in this article to give an interpretation of Verlinde's theory in more accessible terms. This might help other interested scientists to gain a better understanding. It may also evoke reactions from experts, if they would like to oppose the criticism that will come forward. Rather than discussing the underlying fundamentals between thermodynamics and gravity by stringtheoretical descriptions and claims on Shannon's information theory, the focus in this article will be narrowed to a single item. That item is the relationship between Verlinde's expression for the entropy of vacuum and the derived modification of the Newtonian gravitation law such that it matches with Milgrom's empirical law.

The underlying model for these descriptions is a spherical space with radius $L$ (horizon) and a reference screen (holographic screen) with area $A(r)$ at distance $r$ from the centre. In terms of this model, Verlinde's expression for the entropy $S_{D}$ of vacuum is formulated as,

$$
S_{D}(r)=\frac{r}{L} \frac{c^{3} A(r)}{4 G \hbar}
$$

where $G$ is the gravitational constant, $\hbar$ Planck's (modified) constant and $c$ the light velocity in vacuum. From this expression, the gravitational acceleration $g(r)$ is derived as being,

$$
g(r)=g_{N}(r)+g_{D}(r)=g_{N}(r)+\sqrt{\frac{a_{L}}{6} g_{N}(r)} ; a_{L}=\frac{c^{2}}{L} .
$$

where $g_{N}(r)$ is the common Newtonian acceleration and $g_{D}(r)$ an excess term for the contribution due to "apparent dark matter". For large values of $r, g_{N}(r)$ reduces to a small value, such that $g(r)$ approximates the limit value of Milgrom's empirical gravitational law for large $r$, i.e., 
$g(r) \approx \sqrt{a_{0} g_{N}(r)} ; a_{0}=\frac{c^{2}}{6 L}$, if $r \rightarrow \infty$,

where $a_{0}$ is Milgrom's acceleration constant. Identifying this horizon as the Hubble horizon $L=c t_{H}$, where $t_{H} \approx 13.5$ Gyear, it is found that $a_{0} \approx 1.1 \times 10^{-10} \mathrm{~m} / \mathrm{s}^{2}$. This value corresponds with observations on galaxies. This gives a support for the viability of Verlinde's view. To what extent this might be regarded as a decisive one, is the subject of discussion in this article. In this respect, it might be useful to note that, if the horizon would be identified in the sense of the cosmological horizon as defined in the de Sitter model, the horizon would be related with the Cosmological Constant in Einstein's Field Equation [6]. In Verlinde's model the Hubble horizon is equated with the cosmological horizon in a de Sitter space description (compare 1.2 with 2. 10 in [5]).

The article is organized as follows. First, in paragraph 2, the concept behind the formulae (1) and (2). In paragraph 3 a simple derivation of (2) from (1) will be shown and in paragraph 4 the result will be discussed.

\section{The concept}

As pointed out in the introduction, Verlinde's entropy expression for vacuum is inherited from Bekenstein's view on the entropy of a black hole, which has eventually been established as

$S=\frac{A c^{3}}{4 G \hbar}$, where $A=4 \pi R_{S}^{2}$

and where $R_{S}$ is the Schwarzschild radius of the black hole, hence $A$ its peripheral area. This entropy is a measure for the information contained in the black hole. In fact, the entropy is a measure for the number of quantum states available for massive energy within the black hole. For a better understanding of the relationship between the vacuum's entropy and the entropy of a black hole, it might be instructive to visualize the entropy of a black hole in a way as suggested by Susskind [7]. He proposed to consider the entropy $S$ of the black hole as the ratio of its mass $M$ and a sum of $N$ elementary amounts $\Delta M$, brought in or radiated off, by bosons. To this end, the (reduced) Compton wavelength of $\Delta M$ must equate the perimeter $2 \pi R_{S}$ of the black hole, such that

$2 \pi R_{S}=\frac{\hbar}{c \Delta M} \rightarrow \Delta M=\frac{\hbar}{2 c \pi R_{S}}$.

Hence, according to Susskind's interpretation,

$S=N=\frac{M}{\Delta M}=\frac{2 c \pi R_{S}}{\hbar} M=\frac{2 c \pi R_{S}}{\hbar}\left(\frac{2 M G}{c^{2}}\right) \frac{c^{2}}{2 G}=\frac{\pi c^{3}}{\hbar G} R_{S}^{2}=\frac{c^{3}}{4 \hbar G} A$

The result of this simplistic view nicely corresponds with the results of the rigid analysis as originally performed by Hawking [3] and repeated by others [8]. These analyses give the correct answer to the question how to handle the Compton wavelength (reduced or non reduced) and on the correct value of the numerical proportionality factor related with the area $A$ (which was unknown in Bekenstein's conceptual set up). Where the entropy expression for the black hole is an area law without showing the internal entropy distribution, Verlinde's entropy expression (1) for the vacuum basically is a 
volume law. Rather than counting the number of elementary massive amounts $\Delta M$ that are actual present, it counts the number of elementary massive amounts $\Delta M$ that can be maximally comprised by the vacuum together with an heuristic law for the internal distribution. The maximum is bound by the area of the horizon. Entropy is a measure of information, not more, not less. Information presupposes a carrier, being signals or circuits, like in Shannon's case, or massive energies, like in Bekenstein's case. Verlinde's entropy assignment to the vacuum, implicitly presupposes a carrier as well.

To be meaningful, the entropy of the vacuum should be a measure for the amount of possible quantum states of a physical carrier. In Verlinde's case, the carrier could be viewed as imaginary matter. In that respect it is not different from the unknown amount of energy that pops up in the present cosmological standard model, where baryonic matter composes not more than some $5 \%$ of the total gravitational matter in the universe, which, in a way, can be conceived as a sphere with black hole properties, because of simple fact that the Schwarzschild radius $R_{S L}$ of a flat universe with its critical mass density $\rho_{c}$ (if it is naively conceived as a sphere with the observer in the centre) equals the Hubble horizon (if the universe is naively conceived as a sphere with the observer in the centre). This can be easily verified from

$R_{S L}=\frac{2 M G}{c^{2}}=\frac{2 G}{c^{2}}\left(\rho_{c} \frac{4}{3} \pi L^{3}\right)=\frac{8 \pi G}{3 c^{2}} L^{3} \rho_{c}$

Considering the relationship between the critical mass and Hubble's parameter $H_{0}$ at present time,

$\rho_{c}=\frac{3 H_{0}^{2}}{8 \pi G}$ and $H_{0}=\frac{c}{L}$,

it follows readily that $L=R_{S L}$. Therefore, similarly as the entropy of a black hole, one might say that the entropy of this naive model of the universe obeys an area law. By hypothesizing a measure for entropy on the holographic screen at $r$ as an $r / L$ fraction of the entropy at the horizon, Verlinde supposes a uniform distribution of equally sized grains as carriers of entropy within the horizon. By applying Susskind's view (5) on Verlinde's hypothesis (1), the amount $N$ of these carriers on the holographic screen is established as,

$N=S=\frac{r}{L} \frac{c^{3} A(r)}{4 G \hbar}$

The difference $\Delta N$ between the amount of virtual masses on the holographic screen and the screen shifted by an amount $\Delta r$ amounts to

$\Delta N=\frac{r}{L}\left\{\frac{c^{3} A(r+\Delta r)}{4 G \hbar}-\frac{c^{3} A(r)}{4 G \hbar}\right\}=\frac{r}{L} \frac{c^{3}}{4 G \hbar}\left\{4 \pi(r+\Delta r)^{2}-4 \pi r^{2}\right)=2 \pi \frac{r^{2}}{L} \frac{c^{3}}{G \hbar} \Delta r$.

The volume difference $\Delta V$ between the two screens is,

$\Delta V=\frac{4}{3} \pi(r+\Delta r)^{3}-\frac{4}{3} \pi r^{3} \approx 4 \pi r^{2} \Delta r$ 
The volume density of the entropic carriers $\rho_{e}$ therefore amounts to

$\rho_{e}=\frac{\Delta N}{\Delta V}=\frac{c^{3}}{G \hbar} \frac{2 \pi r^{2}}{4 \pi r^{2} L}=\frac{c^{3}}{2 G \hbar L}$

Hence, the volume $V_{g}$ of an entropic grain cell is given as,

$V_{g}=2 \frac{G \hbar L}{c^{3}}$

The supposed existence of those entropy grains is raised by Verlinde to an abstract axiomatic principle. It has to be emphasized, though, that the physical carrier of entropy in the grains is missing.

Let us proceed by reviewing the explicit result in entropic gravity like embodied in the modified Newtonian gravitational acceleration (2). Verlinde claims a match of this result with Milgrom's hypothesis, which is confirmed by a wealth of cosmological observations on galaxies [9]. To my opinion Verlinde's conclusion here is incorrect. Milgrom's equation is different, nl.,

$g=\frac{g_{N}}{\mu(x)}$, with $x=g / a_{0}$ where $\mu(x)=\frac{x}{\sqrt{1+x^{2}}}$.

This implies that, at small $r$, Milgrom's gravitational acceleration coincides with the Newtonian one. This is not true for (2). Evaluation of (14) results into [10],

$g=g_{N} \sqrt{\frac{1+\sqrt{1+4\left(\frac{a_{0}}{g_{N}}\right)^{2}}}{2}} \approx g_{N}\left\{1+\frac{1}{2}\left(\frac{a_{o}}{g_{N}}\right)^{2}\right\}$ as long as $\frac{a_{0}}{g_{N}}<<1$,

and $g \approx \sqrt{a_{0} g_{N}}$ for $\frac{a_{0}}{g_{N}}>>1$.

while entropic gravity cf. (2) predicts

$g=g_{N}\left(1+\sqrt{\frac{a_{0}}{g_{N}}}\right)$.

The difference for $a_{0} / g_{N}<<1$ is so large that one of the two expressions must be wrong. This is particularly clear if applied to the planet orbits in our solar system. The Newtonian gravitational acceleration in Neptune's orbit due to the sun is about $0.6610^{-5} \mathrm{~m} / \mathrm{s}^{2}$. Considering that $a_{0} \approx 1,2 \times 10^{-}$ ${ }^{10} \mathrm{~m} / \mathrm{s}^{2}$, it will be clear that Verlinde's expression can't be correct, because the difference with the Newtonian gravity is too large for being unobserved, like noted by others as well [11]. 


\section{From entropy to dark matter}

Let us consider a sphere in the cosmos. The sphere is filled with an unknown substance. This substance, to be indicated as entropic matter, has a value at distance $r$ from the center as expressed by (1). When a certain amount of baryonic mass $M$ is inserted or withdrawn from the sphere, the value of the entropy at distance $r$ is subject to a change, such that

$$
S_{D}(r)->S_{D}(r)+\Delta S_{D}(r)
$$

where $\Delta S_{D}(r)$ the entropy change to the amount given by the Susskind interpretation as,

$$
\Delta S(r)=\frac{2 \pi r M c^{2}}{\hbar c}
$$

This change of entropy can be interpreted as a volume increase of the sphere that did not contain the mass $M$ before. Insertion of mass means removal of entropy. The removal of entropy cannot be larger than the available amount of it. In other words: the amount of baryonic mass that can be inserted cannot be larger than the amount of enclosed entropic matter. As a consequence, there is a certain boundary $r=R$, depending on the amounted of inserted mass, which divides the space into a domain $r \leq R$ from which entropy can be extracted and a domain $r>R$ from which that is not possible. This latter domain has a constant value for its entropy. Let us characterize, like Verlinde does, the domain $r \leq R$ as elastic and the domain $r>R$ as incompressible. The first domain is characterized by a linear strain-stress characteristic. In the second domain the strain is absent.

Let the volume enclosed within $r$ be $V(r)$ and the volume increase at $r$ due to entropy decrease be $V_{D}(r)$, then we have from (1),

$$
\begin{aligned}
& V(r)=\frac{4}{3} \pi r^{3}=\frac{1}{3} r A(r)=\frac{4 G \hbar L S_{D}(r)}{3 c^{3}}, \text { and } \\
& V(r)+V_{D}(r)=\frac{4 G \hbar L\left\{S_{D}(r)+\Delta S(r)\right\}}{3 c^{3}}=\frac{4 G \hbar L S_{D}(r)}{3 c^{3}}\left\{1+\frac{\Delta S(r)}{S_{D}(r)}\right\} .
\end{aligned}
$$

Hence, under consideration of (18),

$$
V_{D}(r)=\left(\frac{4 G \hbar L}{3 c^{3}}\right)\left(\frac{2 \pi r M c^{2}}{\hbar c}\right) .
$$

Let a strain variable be defined as,

$$
\varepsilon(r)=\frac{V_{D}(r)}{V(r)}
$$

Note that both $V_{D}(r)$ and $V(r)$ are functions of $r$. From (22) we may conclude that the boundary condition is equivalent with

$$
\varepsilon(R)=\frac{V_{D}(R)}{V(R)}=1 .
$$


Hence, from (19), (21) and (23),

$\left(\frac{4 G \hbar L}{3 c^{3}}\right)\left(\frac{2 \pi R M c^{2}}{\hbar c}\right) \frac{1}{4 \pi R^{3} / 3}=\frac{2 G L M}{R^{2} c^{4}}=1 \rightarrow R=\sqrt{\frac{2 G L M}{c^{2}}}$.

Equivalently, $R=\sqrt{L R_{S}} \quad ; R_{S}=\frac{2 M G}{c^{2}}$ (equivalent Schwarzschild radius). Let us proceed by considering the energy due to the strain. Generically,

Energy/Volume $=($ stress $\mathrm{x}$ strain $) / 2$

Because strain is absent for $r>R$, and because the domain $r \leq R$ is considered as being elastic, the energy $W$ due to strain can be expressed as,

$$
W=k_{0} \int_{0}^{R} \varepsilon^{2}(r) A(r) d r=k_{0} V(R) ; \quad V(R)=\int_{0}^{R} \varepsilon^{2}(r) A(r) d r
$$

where $k_{0}$ is proportionality factor, which will appear being irrelevant. Because the quantity $V(R)$ is the volume of the elastic domain, we have from (21),

$\left.V(R)=V_{D}(R)=\frac{4 G \hbar L}{3 c^{3}}\right)\left(\frac{2 \pi R M c^{2}}{\hbar c}\right)$

Differentiation of the integral $V(R)$ after $R$ and change of variable $R \rightarrow r$ gives,

$$
\frac{d V}{d R}=\frac{d V_{D}}{d r}=\varepsilon^{2}(r) A(r)
$$

Hence, under consideration of (21),

$$
\varepsilon^{2}(r)=\frac{1}{A(r)}\left(\frac{4 G \hbar L}{3 c^{3}}\right)\left(\frac{2 \pi M c^{2}}{\hbar c}\right)=\left(\frac{4 G \hbar L}{3 c^{3}}\right)\left(\frac{2 \pi c^{2}}{\hbar c}\right) \sigma_{B}(r) ; \sigma_{B}(r)=\frac{M}{A(r)} .
$$

We may also calculate $\varepsilon(r)$ as a consequence of the subtraction of entropy from the domain $r \leq R$ . From (21) and (22),

$$
\varepsilon(r)=\left(\frac{4 G \hbar L}{3 c^{3}}\right)\left(\frac{2 \pi r M c^{2}}{\hbar c}\right) \frac{1}{4 \pi r^{3} / 3}=\left(\frac{4 G \hbar L}{c^{3}}\right)\left(\frac{2 \pi r M c^{2}}{\hbar c}\right) \frac{1}{\left(4 \pi r^{2}\right) r}=\left(\frac{4 G \hbar L}{c^{3}}\right)\left(\frac{2 \pi c^{2}}{\hbar c}\right)\left(\frac{M}{A(r)}\right)^{*}
$$

Interpretation of this expression requires a careful consideration of its semantics. The quantity $\{M / A(r)\}^{*}$ represents the surface mass density of displaced matter, which is dragged into the elastic sphere, thereby virtually increasing the amount of enclosed mass. Therefore, we may rewrite (29) as 


$$
\varepsilon(r)=\left(\frac{4 G \hbar L}{c^{3}}\right)\left(\frac{2 \pi r c^{2}}{\hbar c}\right) \sigma_{D}(r) ; \sigma_{D}(r)=\left(\frac{M}{A(r)}\right)^{*} .
$$

Hence from (28) and (30),

$$
\left(\frac{4 G \hbar L}{3 c^{3}}\right)\left(\frac{2 \pi c^{2}}{\hbar c}\right) \sigma_{B}(r)=\left\{\left(\frac{4 G \hbar L}{c^{3}}\right)\left(\frac{2 \pi r c^{2}}{\hbar c}\right) \sigma_{D}(r)\right\}^{2} \rightarrow \sigma_{B}(r)=6 \pi\left(\frac{4 G L}{c^{2}}\right) \sigma_{D}^{2}(r) .
$$

Hence, equivalently,

$$
\sigma_{D}(r)=\sqrt{\frac{a_{L}}{24 \pi G} \sigma_{B}(r)} ; a_{L}=\frac{c^{2}}{L}
$$

From this result it is concluded that that next to the surface mass density $\sigma_{B}(r)$ on the holographic screen due to encapsulated baryonic matter, effectively an additional amount of surface mass density $\sigma_{D}(r)$ becomes manifest as a result of virtual entropic matter.

I believe that the analysis (17) -32) covers the methodology, including all considerations that have been put forward by Verlinde in his rather extensive work. Apart from the axiomatic starting point (1), the major ingredients are (a) the split of the spherical domain into an elastic part from which entropy can be subtracted and an incompressible part from which that is not possible, (b) the assessment of the energy that has to be spent if one tries to subtract entropy from the elastic domain and (c) equating the results of the calculation of the strain variable $\varepsilon(r)$ from two perspective angles.

Let us proceed by establishing the relationship between surface mass density and gravitational acceleration. If we have a surface density $\sigma_{B}(r)$ at a spherical area at $r$, the equivalent pointlike mass in the center amounts to

$$
M_{B}=4 \pi r^{2} \sigma_{B}(r) .
$$

This makes a gravitational acceleration $g_{B}(r)$ at $r$ from the equivalence

$$
F=m g_{B}(r)=m \frac{4 \pi r^{2} \sigma_{B}(r) G}{r^{2}} \rightarrow g_{B}(r)=4 \pi G \sigma_{B}(r)
$$

It is tempting to believe that it is possible to assess the gravitational acceleration due to $\sigma_{D}(r)$ as

$$
g_{D}(r)=4 \pi G \sigma_{D}(r)=\sqrt{\frac{16}{24} \pi G a_{L} \sigma_{B}(r)} \rightarrow g_{D}(r)=\sqrt{\frac{a_{L}}{6} g_{B}(r)}
$$

If both contributions, i.e. baryonic matter and dark matter, would behave on par as additive contributions in Poisson's equation, the conclusion from (34) and (35) would confirm (2)

$$
g(r)=g_{B}(r)+g_{D}(r)=g_{N}(r)+\sqrt{\frac{a_{L}}{6} g_{N}(r)} ; g_{N}(r)=g_{B}(r) ; a_{L}=\frac{c^{2}}{L} .
$$


This result corresponds rather well with Milgrom's empirical law for large values of $r$ where $g_{N}<<c^{2} / L$.

\section{Discussion}

Verlinde's model is a spherical bubble with a radius equal to the size of the Hubble horizon. This bubble contains grains to which entropy is assigned as an attribute. Because entropy is not more and not less than a measure of information and because information needs a carrier, the grains are apparently filled with a hypothetical substance, which is not further identified. Instead, the entropy attribute is quid pro quo for the substance. The entropy properties of the bubble and its grains are, inherited from those of a black hole. From an axiomatic hypothesis for an analytical expression of entropy, a theory is developed toward an expression for the gravitational acceleration at a radius $r$ in this sphere. Due to the upper bound in the entropy expression, any amount of baryonic matter inserted in the bubble splits the bubble into an elastic domain and an incompressible domain. As a result, a virtual amount of matter, identified as dark matter, is displaced from the incompressible domain into the elastic domain, thereby virtually increasing the amount of the inserted baryonic matter.

Although a theoretician is free to define an axiomatic hypothesis for his/her theory, the hypothesis is only fruitful if it leads to a theoretical result that is in agreement with empirical evidence. In that respect Verlinde is not convincing, because his conclusion that the theoretical result does so, is incorrect. Furthermore, there are some weak points in the derivation. There is no reason whatsoever why the displaced matter in Poisson's equation can be added on par to the baryonic matter such that the gravitational acceleration is a simple summation of two additive contributions. To the contrary, it is unlikely that this is allowed. Displaced matter has similar characteristics as displaced charge in a Debye process of polarized atomic plasmas. The gravitational equivalent of such a process would include a multiplicative component of dark matter and the gravitational potential in Poisson's equation. Summarizing:

In this article, three major objections have been formulated against Verlinde's theory:

1. The axiomatic quantitative expression for the entropy of the vacuum is heuristic. It lacks a theoretical basis why this heurism should be higher valued than the heurism in Milgrom's expression for the modification of the Newtonian gravitation law.

2. The claim that the result of the theory is in agreement with Milgrom's empirical result is incorrect, because it fails for the spatial range $a_{0} / g_{N}<<1$. Moreover, the seeming match for $a_{0} / g_{N}>>1$, is based upon the heuristic assumption to accept the Hubble scale as the cosmological horizon.

3. The parity of displaced ("dark") matter and baryonic matter in Poisson's equation to arrive at a modified expression for Newton's gravitation law, is a heuristic assumption, which lacks a theoretical basis.

Nevertheless, it is the author's belief that it makes sense to conceive the origin of dark matter as the result of an entropic process. Rather than starting from a new axiomatic hypothesis, one might choose for accepting Einstein's Field Equation with inclusion of the Cosmological Constant as the basis. As is commonly known, a non-trivial viable solution for vacuum requires the presence of a background energy. The format of this energy is akin to that of a perfect fluid. If next to the vacuum an amount of baryonic matter is present, the baryonic matter will disturb the thermodynamic equilibrium state of the fluid. This makes the gravity akin to the Debye process of an electrically charged particle in a plasma. The difference, though, is in the sign of the pressure. In the 
Debye process, the resulting Coulomb field is suppressed ("screened"), while in the gravitational process the field is enhanced. In such a process the gravitational plasma is in an entropic way polarized under influence of the insertion of a baryonic source, thereby giving rise to the gravitational equivalent of displaced charge. The displaced matter is the true origin of dark matter. This elaboration of this alternative view into a full theory can be found in two preprints $[10,11]$.

\section{References}

[1] E. Verlinde, JHEP 1104, 029 ; arXiv: 1001.0785v1 [hep-th] (2011)

[2] J.D. Bekenstein, Phys. Rev. D7, 2333 (1973)

[3] S.W. Hawking, Comm. Math. Phys. 43, 199 (1975)

[4] M. Milgrom, The Astrophysical Journal, 270, 365 (1983)

[5] E. Verlinde, arXiv:1611.02269v2 [hep-th] (2016)

[6] A. Lasenby and C. Doran, Phys. Rev. D, vol.71, Issue 6, id. 063502 (2005)

[7] L. Susskind, Inside blackholes, lecture on youtube (2013)

[8] Qing-yu Cai, Chang-pu Sun and LiYou, Nucl. Phys.B , 905 ,337 ( 2016)

[9] http://www.scholarpedia.org/article/The MOND paradigm of modified dynamics (2017)

[10] E. Roza, doi:10.20944/preprints201705.0164.v3 (2017)

[11] M. Milgrom and R.H. Sanders, arXiv:1612.09582 (2017)

[12] E. Roza, doi:10.20944/preprints201712.0077.v2 (2017) 